\title{
Le $\mathrm{X}^{\mathrm{e}}$ congrès des anthropologues et ethnologues de Russie - Iževsk, juillet 2017
}

\section{Eva Toulouze}

\section{(2) OpenEdition}

\section{Journals}

Édition électronique

URL : https://journals.openedition.org/efo/12861

DOI : 10.4000/efo.12861

ISSN : 2275-1947

Éditeur

INALCO

Référence électronique

Eva Toulouze, «Le Xe congrès des anthropologues et ethnologues de Russie - Iževsk, juillet 2017 », Études finno-ougriennes [En ligne], 49-50 | 2018, mis en ligne le 28 janvier 2019, consulté le 08 juillet 2021. URL : http://journals.openedition.org/efo/12861 ; DOI : https://doi.org/10.4000/efo.12861

Ce document a été généré automatiquement le 8 juillet 2021.

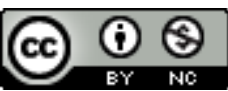

Études finno-ougriennes est mis à disposition selon les termes de la Licence Creative Commons Attribution - Pas d'Utilisation Commerciale 4.0 International. 


\title{
Le $\mathrm{X}^{\mathrm{e}}$ congrès des anthropologues et ethnologues de Russie - Iževsk, juillet 2017
}

\author{
Eva Toulouze
}

Je remercie Aleksi Moine pour ses commentaires pertinents.

1 J'ai rendu compte il y a deux ans du IX congrès, qui avait eu lieu à Ekaterinburg. Je n'avais pas caché ma satisfaction, en raison de la place importante dédiée aux études finno-ougriennes, de l'atmosphère plaisante et constructive et, last but not least, des perspectives qui nous étaient ouvertes par le choix d'Iževsk comme siège pour le congrès suivant, et par l'élection d'Aleksej Zagrebin, le directeur de la filiale oudmourte de l'Académie des sciences, à la présidence de l'association. C'était une occasion pour Iževsk de s'affirmer en tant que capitale scientifique, de montrer son potentiel, de consolider la position de son institut de recherche dans un contexte où, en Russie comme ailleurs, la tendance est à la concentration. J'attendais donc de pied ferme ce congrès dans la capitale oudmourte et je me sentais d'autant plus concernée que j'ai dédié ces dernières années à l'étude des Oudmourtes.

2 J'ai failli ne pas écrire ce compte-rendu. Je n'avais - et je n'ai toujours - aucune envie d'écrire autre chose que de bonnes choses sur «mon» Oudmourtie. Ce sont mes collègues oudmourtes qui m'ont encouragé à ne pas céder à cette tentation. «Il faut que quelqu'un en parle », m'a-t-on dit. Alors je me fais violence.

On comprendra à cette introduction que le congrès ne s'est pas très bien passé. Je vais essayer de rendre compte et d'analyser ce qui s'est déroulé et pourquoi la satisfaction n'a été que mitigée après quelques jours de travaux.

4 En préambule, je sais que les difficultés pratiques avaient été considérables. Iževsk n'avait pas une infrastructure suffisamment développée pour accueillir en même temps 800 personnes, dont un dixième d'étrangers. Il a fallu faire appel à différents établissements d'enseignement et de recherche pour couvrir les besoins en locaux. Mais ce n'est pas là que les problèmes sont apparus. Pour ma part, j'ai résolu le 
problème du logement en louant un appartement situé à égale distance de tous les lieux où auraient lieu mes interventions.

5 L'ouverture a eu lieu en pleine place centrale, au Théâtre d'opéra et de ballet. On peut dire que c'était une ouverture dans l'ensemble réussie. Le chef de l'État oudmourte (par intérim ${ }^{1}$ ), Aleksandr Brečalov, a ouvert les travaux en personne, et à la tribune trônaient toutes les personnalités de l'ethnologie en Russie : le coryphée Valerij Tiškov, Marina Martynova, la responsable de l'ethnologie à l'Académie des sciences, Aleksej Zagrebin, des responsables de NGO, dont Svetlana Smirnova, une ancienne dirigeante oudmourte. J'ai déjà parlé de Zagrebin, mais je dois en parler encore. Quelques mois après Ekaterinburg, il a été élu député à la Douma d'État, position enviable s'il en est et importante pour les Oudmourtes. Dans un entretien, il m'a dit que cela lui donnerait plus de temps pour faire de la recherche, car il ne serait plus accablé par les tâches administratives.

6 Il rêvait. Poutine a lancé une diatribe contre ceux qui veulent être députés et s'accrochent à leurs professions précédentes, n'accomplissant correctement ni l'une ni l'autre de ces tâches. Toujours est-il que Zagrebin continue à rentrer les week-ends à Iževsk et passe les lundis à « son » institut.

7 Les discours d'ouverture sont politiques. Question de nous rappeler que, dans le droit fil des traditions soviétiques, tout est politique et l'intérêt de toute activité est avant tout politique. La science, la recherche, le savoir ne sont jamais des enjeux en tant que tels, mais ils comptent en tant qu'instruments pour obtenir des résultats politiques. Je ne sais pas jusqu'à quel point cette approche est consciente, ou jusqu'à quel point elle est si intériorisée qu'elle n'est même plus objet de réflexion...

Après les discours, une surprise. Nous avions un programme : le troisième jour, une séance plénière était prévue avec une intervention de Zagrebin. Or, on nous annonce qu'elle va avoir lieu sur le champ. Elle sera plus courte que prévu. Elle traite de l'évolution de la recherche dans le domaine finno-ougrien. L'un des thèmes que j'aborde dans mon cours d'initiation aux études finno-ougriennes. Il est bon orateur. Ce qu'il dit est pertinent, je n'apprends rien de très nouveau, mais c'est peut-être réconfortant... Pour moi. Mais bien sûr, la plupart des présents ignorent tout de cette histoire et cette intervention met bien l'accent sur les éléments essentiels du développement des études finno-ougriennes.

9 L'ouverture se termine par un concert. C'est un concert avec plusieurs approches de la musique et de la danse oudmourtes. Il faut louer les organisateurs d'avoir commencé par un chant chanté par une paysanne de mes amies, vivant au village d'Uddjadi (Karamas-Pel'ga de son nom officiel, raïon de Kiasovo), Olga Solovjova, appelée Džakapaj. C'est ce qu'il y a de plus authentique comme production populaire, puisque le chant est présenté sans intermédiaire. Une jeune femme prend le relais, qui chante une chanson avec une technique et une voix typiques de la musique pop. Le groupe emblématique du chant populaire scénique, Ajkaj, est très présent. D’autres groupes les femmes de Tyloburdo par exemple, avec des chants méditatifs - passent eux aussi sur scène. Les spécialistes feront remarquer qu'il n'y a pas grand-chose de nouveau dans les répertoires, mais ne faisons pas la fine bouche. C'est de la bonne qualité, et après tout, peu sont les présents qui ont une connaissance préalable de la culture oudmourte... Le concert s'achève avec deux numéros "patriotiques». Comme si on assistait à une fête de la république... L'un est chanté par Bogdan Anfinogenov Superudmurt (https://www.youtube.com/watch?v=XrXQqwXo4nc), une sorte de rap 
accumulant des mots-référence, entre autres toponymes, en oudmourte mais aussi en russe, sur un rythme endiablé. Pas ma tasse de thé, mais je suis heureuse que cela existe. La multiplicité des genres est cruciale pour le maintien en bonne santé d'une culture. Par ailleurs, Bogdan est historien, doctorant et il s'apprête à finir une thèse sur l'ethnofuturisme. Ainsi l'idée derrière ce programme, c'est bien de montrer la diversité des manifestations de l'oudmourtitude et je pense que cela s'est avéré pertinent.

Les activités scientifiques commencent l'après-midi même. Il n'y a à l'évidence pas de "délégation » française, et les deux Français présents ne représentent pas, ou pas uniquement, des institutions françaises. Mon voyage et, de manière générale, ma mission sont couverts par l'université de Tartu, et Aleksi Moine n'a d'autre rattachement officiel, au moment du congrès, que l'université de Reykiavik, où il vient de terminer un master. Pour ma part, je passerai mes journées dans les sections où j'interviens, la section d'anthropologie visuelle le premier jour et celle sur les religions comparées le deuxième. Aleksi m'accompagnera le premier jour et passera le deuxième dans la section Mythologie et Folklore, où il intervient.

Tout commence bien. Evgenij Aleksandrov, responsable de la section d'anthropologie visuelle, est un vieil ami, un habitué du festival Worldfilm de Tartu que j'organise. À Ekaterinburg, les organisateurs n'avaient pas accepté sa section : je ne pense pas être trop loin de la vérité en suggérant que le fait que l'organisateur principal du congrès fût Andrej Golovnev, lui aussi réalisateur et animateur de festival avait joué un certain rôle dans la décision d'écarter Evgenij. De ce point de vue-là, pas de problèmes cette année. Nous sommes deux à intervenir sur le domaine finno-ougrien, plus précisément oudmourte, Elena Popova et moi. Elena parle de la dimension visuelle d'une exposition qu'elle a contribué à organiser au musée de la ville d'Iževsk sur les cours intérieures de la ville. Pour ma part, je parle des cérémonies animistes oudmourtes au Bachkortostan et de la manière de les filmer. En principe, c'est une intervention commune avec Liivo Niglas, mais Liivo ne s'est pas déplacé. L'ensemble des autres interventions sont de bon niveau et présentées de manière vivante. C'est d'ailleurs l'une des choses qui m'auront impressionnée à ce congrès : traditionnellement la présentation n'est pas le point fort des collègues de Russie, qui ont (avaient?) souvent tendance à lire machinalement un texte écrit, avec l'objectif d'arriver le plus vite possible à sa fin. Rien de tel dans les deux sections que j'ai fréquentées cette année. C'est là une évolution bienvenue. Ceci dit, les échos que j'ai d'autres sections soulignent que cette pratique se poursuit, notamment parce qu'il fallait très vite terminer le programme, et parfois les intervenants n'ont eu à leur disposition que quinze minutes.

12 En même temps, ce premier jour, je suis déçue. J'étais intéressée par deux interventions qui traitaient des Oudmourtes dans la section Mythologie et folklore, celle de Tatjana Minniahmetova, dont le matériau provient de la communauté que j'étudie, et celle de Nikolaj Anisimov, l'un de mes plus proches collaborateurs. Pas de chance : ils interviennent en même temps que moi. Eux sont contents, débarrassés de leur pensum, mais moi je regrette de n'avoir pas pu les écouter. C'est que les sections parlant de sujets analogues sont toutes en même temps. Erreur évitable? Je ne sais pas.

13 Le deuxième jour je suis intégrée dans une autre section. Animée par Evgenij Prigorin (Ukraine) et par mon amie proche Elena Danilko, spécialiste des Vieux-Croyants, cette section consacrée à l'étude des religions m'est bien connue, car je m'y étais inscrite à Ekaterinburg et que je l'avais vraiment appréciée. C'est pour cela que j'ai proposé, cette année encore, d'y faire ma deuxième intervention - avec Laur Vallikivi celle-ci - 
elle aussi sur les pratiques religieuses oudmourtes au Bachkortostan. Comme la dernière fois, les interventions sont toutes de qualité. Mais bien sûr, ily a un problème inhérent à ce genre de congrès. Beaucoup de chercheurs se sont inscrits, ont envoyé leurs résumés et ont été acceptés, ils figurent sur le programme. Mais tous ne viendront pas sur place. Ainsi, les responsables de section, même s'ils ont fait une enquête à titre privé, ne savent jamais réellement sur combien de contributions compter. La communication entre les organisateurs du congrès, qui suivent les arrivées réelles, et les responsables de section est inexistante. Autrement, en début d'après-midi, les responsables de section reçoivent bien une information inattendue : l'assemblée générale de l'Association des Anthropologues et ethnologues de Russie est déplacée et aura lieu le jour-même à 17 heures. Les responsables des sections sont invités à terminer leurs travaux plus tôt pour pouvoir y assister. Cela veut dire que beaucoup de personnes, dans ma section, ne pourront pas intervenir. Et c'est dommage aussi pour l'assemblée: tous ne pourront pas yassister, car certaines sections continuent leurs travaux.

Cette année, je passe le plus clair de mon temps avec les Oudmourtes, avec mes camarades de terrain, Nikolaj, Ranus Sadikov et Denis Kornilov. Le soir, nous nous sommes inscrits pour une soirée payante (mais pas chère) appelée Ethnoparty, qui a lieu à la « Vallée des castors ", un bel endroit en ville, mais suffisamment isolé pour qu'on s'y sente comme à la campagne. Nous avons décidé d'y faire une réunion rassemblant les participants à mon projet IUF, dont beaucoup sont présents - ne sont absents que les Estoniens Liivo Niglas et Laur Vallikivi et une collègue linguiste du Bachkortostan, Anna Bajdullina (qui nous rejoindra le lendemain). Je n'ai pas vraiment le temps d'explorer tous les lieux. Il y a un bar, les berges d'une rivière, un espace avec des tables et des chaises, une petite scène, des espaces de tissage (on peut apprendre à tisser) et d'exposition (avec des robes de Mademoiselle Oudmourte, l'initiative de mode de Darali Leli) et des bâtiments en bois, d'apparence un peu kitsch, mais aménagés avec beaucoup de goût à l'intérieur. Ils comprennent quelques chambres (certains participants estoniens y sont logés), des saunas, un restaurant, des espaces de socialisation. C'est là que nous tenons notre réunion. Ensuite nous rejoignons l'ensemble des participants. Une danse endiablée mélangeant participants et professionnels remplit la scène. Ensuite interviennent deuxjeunes filles et un garçon, Evgenij Bikuzin, (qui avait participé aux journées oudmourtes à Paris) qui pratiquent un folklore en même temps authentique et légèrement décalé. On peut déguster des spécialités oudmourtes confectionnées en partie devant nous, et du jus d'argouse (pour ceux qui n'ont pas envie de bière). C'est un moment de socialisation. Un DJ passe de la musique oudmourte sur laquelle beaucoup dansent.

La soirée ne s'arrêtera pas là. Le groupe dans lequel je me trouve décide de continuer ailleurs. On marche donc un peu en ville, nous avançons sous la direction de Darali Leli. Elle me paraît être le meilleur exemple de culture oudmourte urbaine. Elle est née en ville, et est résolue à y porter la culture oudmourte, ou plutôt à en façonner une qui soit satisfaisante pour cette catégorie de personnes et pour ses besoins. Elle me semble réussir de manière admirable. Nous sommes tout un groupe, où Aleksi, Tatjana Alybina (une doctorante marie de Tartu) et moi sommes les seuls non oudmourtes. Nous nous installons dans un café que l'un de nous connaît bien (et où il est connu), et on nous donne la salle des VIP. On peut y manger et surtout y boire. L'un de nos compagnons s'éclipse, pour revenir un quart d'heure plus tard avec son accordéon. Ainsi se passera cette soirée, qui se terminera vers quatre heures du matin : accordéon, chant, danses. 
C'est ainsi que les Oudmourtes, y compris les jeunes urbains, se détendent. Tout le monde dansera et je constate que c'est là un bon antidote à la boisson. Boire du thé et danser : tel est le secret des Oudmourtes pour supporter la consommation d'alcool qui est dans les mœurs. Une soirée réellement intéressante et édifiante.

Certes, nous savions que nous n'allions pas être frais le lendemain matin, mais ce n'est pas grave: la matinée, dans le programme, est dédiée à la conférence plénière de Zagrebin... qui a déjà eu lieu. A déjà eu lieu aussi l'assemblée générale de l'association. Nous avons donc décidé de récupérer nos forces dans la matinée et de nous rendre à une section l'après-midi. Nous choisissons la section consacrée aux peuples de la Volga, animée par notre collègue tchouvache Ekaterina Jagafova. Cela tombe bien, car elle a lieu au musée ethnographique Kuzebaj Gerd, où à 19 heures je montre, dans le cadre du congrès, mais en projection ouverte, un film de Liivo Niglas sur une cérémonie animiste de village chez les Oudmourtes du Bachkortostan. En assistant à la session sur la Volga, nous allions nous trouver sur place pour faire toutes les vérifications indispensables et visiter le musée. Si ce n'est qu'en arrivant au musée, nous découvrons avec stupeur que la session n'a pas lieu. Ils ont terminé les travaux hier soir, nous dit-on. Cela nous permet, certes, de visiter le musée, mais cela laisse un arrière-goût amer dans la bouche. Nous apprendrons que la plupart des sessions ont été conclues la veille, laissant au congrès un jour et demi pour les activités scientifiques. Seules quelques sessions, par exemple celle consacrée à la muséologie et tenue au skanzen de Ludorvaj, ont profité de leur temps pour rallonger le temps de parole des participants et permettre des discussions.

17 Nous avons le temps de visiter le musée - après que j'ai remis le disque dur où se trouve le film aux techniciens. Ce n'est pas la première fois que je le visite, mais la précédente datait de 1994... Je tenais donc à le visiter à nouveau. C'est bien sûr la partie consacrée aux Oudmourtes qui m'intéresse, plus que les autres, dédiées à l'histoire du territoire histoire qui n'a pas été animée par la population autochtone mais par le colonisateur. C'est cette partie-là qui accueille le visiteur et... et je suis déçue. Non pas bien sûr par les matériaux eux-mêmes, qui sont splendides, mais par la conception qui régit leur présentation. L'idée de construire des saynètes n'est pas mauvaise en soi. Elle caractérise manifestement les musées conçus à l'époque soviétique, comme le musée ethnographique de Saint-Pétersbourg. Le problème est que ces saynètes reposent sur une conception en même temps essentialiste et surannée de la communauté à laquelle l'exposition est dédiée. Dans " une noce oudmourte» on va trouver des personnages revêtant des costumes de régions différentes. C'est-à-dire que ce qui est recherché, ce n'est pas une vraisemblance ethnographique, mais un prétexte pour montrer de jolies choses. On va nous présenter les « Oudmourtes » en ignorant totalement les différences locales entre les groupes, qui enracinent la culture dans un continuum riche en variations. C'est ma connaissance (en réalité superficielle) du costume oudmourte qui me permet de situer ce qui nous est montré, sans la moindre aide de la part du musée. J'ai rencontré ce même défaut au musée de Saint-Pétersbourg. Là, aucune différence n'est faite entre Nénetses de la toundra et Nénetses des forêts, et le premier objet que le visiteur aperçoit pour illustrer la culture komie, c'est un traîneau, objet qui ne caractérise qu'un groupe marginal de Komis, les éleveurs de rennes de l'Ižma, et qui n'est nullement généralisable à l'ensemble des Komis. Les explications n'illuminent pas le visiteur... Ici, une prière est affichée sur la paroi d'une saynète. On le comprendra, je suis intéressée, car l'un des objectifs de mon projet IUF est bien de publier un recueil de prières. Nous avons bien le texte de la prière. Mais d'où provient-elle? De quelle 
région? Par qui a-t-elle été récitée, collectée ? En quelle année ? Rien. Du coup, on a l'impression que le musée nous livre quelque chose d'inhérent aux Oudmourtes, de transcendant, nous privant de tout enracinement dans l'espace et dans le temps. Je suis d'autant plus peinée que le musée d'histoire d'Iževsk, pour sa part, est riche d'inventivité et de rigueur scientifique, sans que cela le rende ennuyeux ou pédant pour le visiteur béotien. Pourquoi ne pas consacrer la même attention créative aux autochtones de ces terres? Je ne veux pas mettre en cause la direction actuelle du musée, qui essaye de se montrer active. Mais faute de spécialistes, d'anthropologues aguerris, ils en restent à une conception muséographique du $\mathrm{XIX}^{\mathrm{e}}$ siècle, alors que les besoins évoluent et les approches scientifiques aussi. La prise en compte, dans l'ethnographie et dans le folklore oudmourtes, du facteur local, est une nécessité criante.

18 La projection de notre film attire plus d'une centaine de personnes. Nous présentons, Ranus et moi, les circonstances de ce tournage et les cérémonies oudmourtes. Le film est inachevé en réalité, nous avons encore à travailler sur les sous-titres. Dans le public, peu d'ethnographes, mais les personnes actives dans la pratique religieuse oudmourte comme les vös'as' de la république, Albert Razin, Andrej Perevoščikov - et beaucoup d'Oudmourtes, dont certains que je n'avais pas vus depuis des décennies. Une soirée satisfaisante et qui devra être répétée. Une manière de rendre aux Oudmourtes tout ce qu'ils nous livrent.

19 Le congrès est presque terminé, pensons-nous. Quand nous prenons congé de nos amis spécialistes de la région de la Volga, Elena Popova et Aleksandr Černyh (de Perm'), nous leur disons allègrement "à demain »... pour apprendre, par Černyh que la séance conclusive est annulée. Il est bien placé dans les milieux de l'ethnologie russe, on peut lui faire confiance. Du coup, nous évitons la déception de bien des participants, qui se rendront le lendemain matin au Théâtre pour trouver les portes fermées et se confronter à des huissiers étonnés qui savent seulement qu'il ne se passera rien ce jour-là...

Bien sûr, c'est la cerise sur le gâteau. Un rassemblement sans conclusion, sans occasion donnée aux participants, qui ont été entre-temps éparpillés entre les sections, de se retrouver. Pourquoi? La raison est simple, c'est elle qui a motivé depuis le début les changements de programme. Les personnes les plus haut placées, celles qui composent la tribune, ont un autre engagement à Moscou et tout le monde est parti. Il n'y a personne à mettre à la tribune, donc on annule. N'aurait-on pas pu profiter de l'occasion pour former une tribune avec les chercheurs de l'institut d'Iževsk?

21 Ici apparaît une autre faiblesse de l'organisation de ce congrès : les chercheurs locaux ont été ignorés. Le congrès a été organisé par des gens qui ne connaissent pas Iževsk et dont la motivation n'était manifestement pas de valoriser la recherche locale. Le remplaçant de Zagrebin, ou plutôt celui qui occupe les fonctions de directeur, Aleksandr Egorov, n'a même pas été invité à la tribune le premier jour. Il est réduit à une position d'homme de paille, qui, aux dires des chercheurs de l'institut, ne peut décider de rien sans l'aval de Zagrebin et donc n'a aucune chance de grandir dans sa fonction. C'est vraiment un cadeau empoisonné qui lui a été fait...

22 Nous ne perdrons pas complètement notre journée : nous allons à l'institut regarder l'exposition-vente de livres, puis au musée d'Iževsk où Elena Popova nous organise une visite, nous regardons Iževsk du haut de la grande roue (sous la pluie cependant) et nous terminons la journée par une soirée «conclusive» alternative chez 
Jurij Perevožčikov, lui-même ethnologue (et responsable de l'Agence pour la protection du patrimoine), qui a invité quelques chercheurs travaillant sur la région de la Volga on déguste des spécialités, on va au sauna. Au moins pour quelques-uns d'entre nous, il $\mathrm{y}$ aura un point final.

Au-delà de la petite histoire, l'arrière-goût d'amertume demeure. Dans une période où l'existence d'institutions locales de l'Académie des sciences est menacée en faveur d'une centralisation croissante, Iževsk avait une occasion de se montrer comme une véritable capitale de la recherche dans la zone Volga-Oural. Non pas qu'elle pouvait espérer détrôner Ekaterinburg, mais au moins essayer de garder son institut, de lui donner une occasion de faire ses preuves.

En même temps, tout est inquiétant. Les programmes doctoraux ont été fermés, les chercheurs meurent et ne sont pas remplacés, on ne voit pas à l'horizon poindre une génération d'ethnologues et de folkloristes pour prendre la relève. Cette année, une seule place de doctorant est prévue pour toutes les sciences humaines... Les jeunes qui s'affirment et qui seraient un espoir ne sont pas avidement recrutés par les institutions oudmourtes et font bénéficier autrui de leur talent: Anisimov, par exemple, a été recruté avant même la soutenance de son doctorat par le musée Littéraire estonien de Tartu, qui s'est empressé de s'assurer un cadre de valeur. Et après tout, pour le jeune chercheur, c'est la meilleure solution. Pourquoi se retrouver immobilisé dans un contexte où tout travail de terrain est interdit faute de financement? Où les archives sont dans un état de chaos déplorable, alors même que l'archiviste part en congé maternité et qu'on se demande encore si elle va être remplacée?

Je suis désolée de devoir produire un texte relevant tous ces problèmes. Pourtant, cela ne mène nulle part de céder à la politique de l'autruche. Il est important pour nous d'avoir des partenaires oudmourtes avec qui discuter de mes travaux. Il n'est pas juste de laisser le terrain à d'autres, faute de moyens pour travailler. Si les chercheurs oudmourtes n'arrivent pas à faire entendre leur voix maintenant, cela risque de durer longtemps, car bientôt, il n'y aura plus personne pour prendre leur relève.

\section{NOTES}

1. Le chef d'État nommé, Solovjov, un Oudmourte, a été démis et arrêté, accusé de corruption, au mois d'avril 2017. Son remplaçant a été nommé par le Kremlin, et pendant que j'étais en Oudmourtie, Vladimir Poutine s'y est rendu pour confirmer sa position avant les élections de septembre. 


\section{INDEX}

Mots-clés : anthropologues, congrès, recherche scientifique, anthropologie, oralité, xxie siècle début, Oudmourtes, Bachkortostan, Ekaterinburg, Iževsk, Karamas-Pel'ga, Ludorvaj, Moscou, Oudmourtie, Perm', Reykjavik, Russie, Saint-Pétersbourg, Tartu, Ukraine, Volga

Keywords : anthropologists, congress, scientific research, Anthropology, Folklore, 21th century beginning, Udmurt, Bashkortostan, Izhevsk, Karamas-Pel'ga, Ludorvay, Moscow, Perm', Reykjavik, Russia, Saint-Petersburg, Tartu, Udmurtia, Ukraine, Volga, Yekaterinburg motscleset antropoloogid, kongress, teadusuuringud, antropoloogia, rahvaluule, 21. sajand algus, udmurdid, Baškortostan, Iževsk, Karamas-Pel'ga, Ludorvaj, Perm', Moskva, Reykjavik, Sankt-Peterburg, Tartu, Udmurdimaa, Ukraina, Venemaa, Volga, Jekaterinburg 